\title{
VECTOR METRIC SPACES AND SOME PROPERTIES
}

\author{
Cüneyt ÇeviK - Ishak Altun
}

\begin{abstract}
In this paper we introduce vector metric spaces and we give some properties of this spaces. Also we prove Baire theorem and Banach fixed point theorem on this spaces.
\end{abstract}

\section{Introduction}

Let $E$ be a Riesz space with the positive cone $E_{+}=\{x \in E: x \geq 0\}$. If every non-empty subset of $E$ which is bounded above has a supremum, then $E$ is called Dedekind complete. If $\left(a_{n}\right)$ is a decreasing sequence in $E$ such that $\inf a_{n}=a$, we write $a_{n} \downarrow a$. $E$ is said to be Archimedean if $n^{-1} a \downarrow 0$ holds for every $a \in E_{+}$. A sequence $\left(b_{n}\right)$ is said to order convergent (or $o$-convergent) to $b$ if there is a sequence $\left(a_{n}\right)$ in $E$ satisfying $a_{n} \downarrow 0$ and $\left|b_{n}-b\right| \leq a_{n}$ for all $n$, and written $b_{n} \stackrel{o}{\rightarrow} b$ or $o$-lim $b_{n}=b$, where $|a|=a \vee(-a)$ for any $a \in E$. Furthermore $\left(b_{n}\right)$ is said to be order-Cauchy (or $o$-Cauchy) if there exists a sequence $\left(a_{n}\right)$ in $E$ such that $a_{n} \downarrow 0$ and $\left|b_{n}-b_{n+p}\right| \leq a_{n}$ holds for all $n$ and $p$. $E$ is said to be $o$-Cauchy complete if every $o$-Cauchy sequence is $o$-convergent. For notations and other facts regarding Riesz spaces we refer to [1].

Huang and Zhang [3], defined cone metric space and proved some fixed point theorems for contractive mappings on this spaces. Let $X$ be a non-empty set. The cone metric on $X$ take value on an ordered Banach space. On the other

2000 Mathematics Subject Classification. Primary 47B60; Secondary 54H25.

Key words and phrases. Vector metric space, Riesz space, contractive mapping, fixed point.

(C)2009 Juliusz Schauder Center for Nonlinear Studies 
hand, Zabreı̌ko [5] defined $K$-metric space and $K$-normed space. Also Zabreǔko gave some fixed point theorems with linear and nonlinear Lipschitz conditions on $K$-metric spaces. The $K$-metric space on $X$ take value on an ordered linear space $B$ with a cone $K$. In this paper we introduce vector metric spaces. A vector metric space on $X$ take value on a Riesz space.

We replace the real numbers by Riesz space and define vector metric spaces $(X, d, E)$. In Section 2, we define basic concepts of the metric spaces theory in vector metric spaces. Then we obtain some new results and give the Baire's Theorem in the vector metric spaces.

In Section 3, we give a proof of Banach fixed point theorem on vector metric spaces. Our results are generalization of some fixed point theorems in metric spaces [2], [4].

\section{Vector metric spaces}

In this section we define vector metric spaces and prove some properties.

Definition 2.1. Let $X$ be a non-empty set and $E$ be a Riesz space. The function $d: X \times X \rightarrow E$ is said to be a vector metric (or E-metric) if it is satisfying the following properties:

(a) $d(x, y)=0$ if and only if $x=y$,

(b) $d(x, y) \leq d(x, z)+d(y, z)$

for all $x, y, z \in X$. Also the triple $(X, d, E)$ (briefly $X$ with the default parameters omitted) is said to be vector metric space.

It is obvious that vector metric spaces generalize metric spaces.

For arbitrary elements $x, y, z, w$ of a vector metric space, the following statements are satisfied:

(i) $0 \leq d(x, y)$;

(ii) $d(x, y)=d(y, x)$;

(iii) $|d(x, z)-d(y, z)| \leq d(x, y)$;

(iv) $|d(x, z)-d(y, w)| \leq d(x, y)+d(z, w)$.

Now we give some examples of vector metric spaces.

ExAmple 2.2. (a) A Riesz space $E$ is a vector metric space with $d: E \times E \rightarrow$ $E$ defined by

$$
d(x, y)=|x-y|
$$

This vector metric is called to be absolute valued metric on $E$.

(b) It is well known that $\mathbb{R}^{2}$ is a Riesz space with coordinatwise ordering defined by

$$
\left(x_{1}, y_{1}\right) \leq\left(x_{2}, y_{2}\right) \quad \text { if and only if } \quad x_{1} \leq x_{2} \text { and } y_{1} \leq y_{2}
$$


for $\left(x_{1}, y_{1}\right),\left(x_{2}, y_{2}\right) \in \mathbb{R}^{2}$. Again $\mathbb{R}^{2}$ is a Riesz space with lexicographical ordering defined by

$$
\left(x_{1}, y_{1}\right) \leq\left(x_{2}, y_{2}\right) \quad \text { if and only if } x_{1}<x_{2} \text { or } x_{1}=x_{2}, y_{1} \leq y_{2}
$$

Therefore $d: \mathbb{R}^{2} \times \mathbb{R}^{2} \rightarrow \mathbb{R}^{2}$ defined by

$$
d\left(\left(x_{1}, y_{1}\right),\left(x_{2}, y_{2}\right)\right)=\left(\alpha\left|x_{1}-y_{1}\right|, \beta\left|x_{2}-y_{2}\right|\right)
$$

is a vector metric, where $\alpha, \beta$ are positive real numbers.

(c) Let $d: \mathbb{R} \times \mathbb{R} \rightarrow \mathbb{R}^{2}$,

$$
d(x, y)=(\alpha|x-y|, \beta|x-y|)
$$

where $\alpha, \beta \geq 0$ and $\alpha+\beta>0$. Then $d$ is a vector metric with coordinatwise or lexicographical ordering.

REMARK 2.3. Note that $\mathbb{R}^{2}$ is Archimedean with coordinatwise ordering but not with lexicographical ordering.

Now we give some definitions.

Definition 2.4. (a) A sequence $\left(x_{n}\right)$ in a vector metric space $(X, d, E)$ vectorial converges (or E-converges) to some $x \in E$, written $x_{n} \stackrel{d, E}{\longrightarrow} x$, if there is a sequence $\left(a_{n}\right)$ in $E$ satisfying $a_{n} \downarrow 0$ and $d\left(x_{n}, x\right) \leq a_{n}$ for all $n$.

(b) A sequence $\left(x_{n}\right)$ is called E-Cauchy sequence whenever there exists a sequence $\left(a_{n}\right)$ in $E$ such that $a_{n} \downarrow 0$ and $d\left(x_{n}, x_{n+p}\right) \leq a_{n}$ holds for all $n$ and $p$.

(c) A vector metric space $X$ is called $E$-complete if each $E$-Cauchy sequence in $X E$-converges to a limit in $X$.

(d) A subset $Y$ of a vector metric space $X$ is said to be $E$-closed whenever $\left(x_{n}\right) \subseteq Y$ and $x_{n} \stackrel{d, E}{\longrightarrow} x$ imply $x \in Y$.

Using the above definitions, we have the following properties:

If $x_{n} \stackrel{d, E}{\longrightarrow} x$, then

(i) The limit $x$ is unique.

(ii) Every subsequence of $\left(x_{n}\right) E$-converges to $x$.

(iii) If also $y_{n} \stackrel{d, E}{\longrightarrow} y$, then $d\left(x_{n}, y_{n}\right) \stackrel{o}{\rightarrow} d(x, y)$.

The relationships between the concepts of boundedness and diameter of a subset of a vector metric space are different from the usual. For a non-empty subset $A$ of a vector metric space $X$ its $E$-diameter defined by $d(A)=\sup \{d(x, y)$ : $x, y \in A\}$ if $\sup \{d(x, y): x, y \in A\}$ in $E$. Furthermore, if there exists an $a>0$ in $E$ such that $d(x, y) \leq a$ for $x, y \in A$, then $A$ is called $E$-bounded set. If $E$ is Dedekind complete, then every $E$-bounded set of $X$ has a diameter. 
TheOREM 2.5. For the vector metric space $(X, d, E)$ the followings hold:

(a) Every E-convergent sequence is an E-Cauchy sequence;

(b) Every E-Cauchy sequence is E-bounded;

(c) If an E-Cauchy sequence $\left(x_{n}\right)$ has a subsequence $\left(x_{n_{k}}\right)$ such that

$$
x_{n_{k}} \stackrel{d, E}{\longrightarrow} x \text { then } x_{n} \stackrel{d, E}{\longrightarrow} x
$$

(d) If $\left(x_{n}\right)$ and $\left(y_{n}\right)$ are E-Cauchy sequences, then $\left(d\left(x_{n}, y_{n}\right)\right)$ is an oCauchy sequence.

Proof. (a) Let $x_{n} \stackrel{d, E}{\longrightarrow} x$ in $X$. Since there exists a sequence $\left(a_{n}\right)$ in $E$ such that $a_{n} \downarrow 0$ and

$$
d\left(x_{n}, x_{n+p}\right) \leq d\left(x_{n}, x\right)+d\left(x_{n+p}, x\right) \leq a_{n}+a_{n+p} \leq 2 a_{n}
$$

for all $n$ and $p$, then $\left(x_{n}\right)$ is an $E$-Cauchy sequence in $X$.

(b) Let $\left(x_{n}\right)$ be an $E$-Cauchy sequence in $X$. Since there exists a sequence $\left(a_{n}\right)$ in $E$ such that $a_{n} \downarrow 0$ and $d\left(x_{n}, x_{n+p}\right) \leq a_{n}$ for all $n$ and $p$, then $d\left(x_{n}, x_{n+p}\right)$ $\leq a_{1}$, that is, $\left(x_{n}\right)$ is $E$-bounded in $X$.

(c) Let $\left(x_{n}\right)$ be an $E$-Cauchy sequence and let $\left(x_{n_{k}}\right)$ be a subsequence of $\left(x_{n}\right)$ such that $x_{n_{k}} \stackrel{d, E}{\longrightarrow} x$ in $X$. If we take $n_{k}=n+p$, where $n \leq n_{k}$ for all $n$, then there exist two sequences $\left(a_{n}\right)$ and $\left(b_{n}\right)$ in $E$ such that $a_{n} \downarrow 0, b_{n} \downarrow 0$ and

$$
d\left(x_{n}, x\right) \leq d\left(x_{n}, x_{n+p}\right)+d\left(x, x_{n+p}\right) \leq a_{n}+b_{n+p} \leq a_{n}+b_{n},
$$

hence $x_{n} \stackrel{d, E}{\longrightarrow} x$.

(d) Since there exist two sequence $\left(a_{n}\right)$ and $\left(b_{n}\right)$ in $E$ such that $a_{n} \downarrow 0, b_{n} \downarrow 0$ and

$$
\left|d\left(x_{n}, y_{n}\right)-d\left(x_{n+p}, y_{n+p}\right)\right| \leq d\left(x_{n}, x_{n+p}\right)+d\left(y_{n}, y_{n+p}\right) \leq a_{n}+b_{n}
$$

for all $n$ and $p$, then the sequence $\left(d\left(x_{n}, y_{n}\right)\right)$ is an $o$-Cauchy sequence in $E$.

When $E=\mathbb{R}$, the concepts of vectorial convergence and convergence in metric are the same. When also $X=E$ and $d$ is the concepts of absolute valued vector metric, vectorial convergence and convergence in order are the same. When $E=\mathbb{R}$, the concepts of $E$-Cauchy sequence and Cauchy sequence are the same.

Now, let us fix a vector metric space $(X, d, E)$. For two elements $a$ and $b$ in $E$, we shall write $a<b$ to indicate that $a \leq b$ but $a \neq b$, while $b>a$ stands for $a<b$. If $x \in X$, then the open ball at $x$ with radius $r>0$ in $E$ is the set $B(x, r)=\{y \in X: d(x, y)<r\}$. Now, the open subsets of $X$ can be defined in the usual way. A subset $A$ of $X$ is called open if for every $x \in A$, there exists some $r>0$ in $E$ such that $B(x, r) \subseteq A$. Every open ball $B(x, r)$ is an open set. 
The collection of open subsets $\tau_{d, E}$ is a topology on $X$, called the vector metric topology.

Definition 2.6. Let $(X, d, E)$ be a vector metric space.

(a) A subset $Y$ of $X$ is called $\tau_{d, E}$-dense whenever $B(x, r) \cap Y \neq \emptyset$ for each $x \in X$ and $0<r \in E$.

(b) A subset $Y$ of $X$ is called $E$-dense whenever for every $x \in X$ there exists a sequence $\left(x_{n}\right)$ in $Y$ satisfying $x_{n} \stackrel{d, E}{\longrightarrow} x$.

We have already following result.

Corollary 2.7. Let $Y$ be a subset of a vector metric space $(X, d, E)$ with E Archimedean. If $Y$ is $\tau_{d, E}$-dense, then $Y$ is E-dense.

We will use some properties of vector metric topology for constructing to the Baire's theorem . The following result explain the Cantor intersection theorem for vector metric spaces.

THEOREM 2.8. Let $X$ be a E-complete vector metric space such that $E$ is Dedekind complete. If a decreasing sequence of non-empty E-closed subsets has vanishing diameter in $X$, then the intersection of the sequence is a singleton.

ProOF. Let $\left(F_{n}\right)$ be a decreasing sequence of non-empty $E$-closed subsets of the $E$-complete vector metric space $X$ where $E$ Dedekind complete, and assume $\lim _{n \rightarrow \infty} d\left(F_{n}\right)=0$. The intersection $F=\bigcap_{n=1}^{\infty} F_{n}$ cannot have more than one point, for if $x, y \in F$, then $d(x, y) \leq d\left(F_{n}\right)$ for each $n$, so $d(x, y)=0$, which implies $x=y$.

To see that $F$ is non-empty, for each $n$ pick some $x_{n} \in F_{n}$. Since $d\left(x_{n}, x_{n+p}\right)$ $\leq d\left(F_{n}\right)$ for each $n$ and $p$, the sequence $\left(x_{n}\right)$ is $E$-Cauchy. Since $X$ is $E$-complete there is some $x \in X$ with $x_{n} \stackrel{d, E}{\longrightarrow} x$. But $x_{n+p}$ belongs to $F_{n}$, and each $F_{n}$ is $E$-closed, so $x$ belongs to $F_{n}$ for each $n$.

Now we give Baire Theorem for vector metric spaces.

THEOREM 2.9. A E-complete vector metric space $X$ is a Baire space whenever $E$ Archimedean and Dedekind complete.

Proof. Let $X$ be a $E$-complete vector metric space with $E$ Archimedean. Now let $\left(A_{n}\right)$ be a sequence of $\tau_{d, E}$-dense open subsets of $X$ and put $A=$ $\bigcap_{n=1}^{\infty} A_{n}$. It is sufficient to show that $A$ is a $\tau_{d, E}$-dense subset of $X$, or that $B(x, r) \cap A \neq \emptyset$ for each $x \in X$ and $r>0$. So fix $x \in X$ and $r>0$.

Since $A_{1}$ is open and $\tau_{d, E}$-dense in $X$, there exist $y_{1} \in X$ and $0<r_{1}<a$ where $a$ is some element of $E$ such that $C\left(y_{1}, r_{1}\right) \subset B(x, r) \cap A_{1}$, where $C(x, r)$ denotes the closed ball of radius $r$ centered at $x$. Similarly, since $A_{2}$ is open

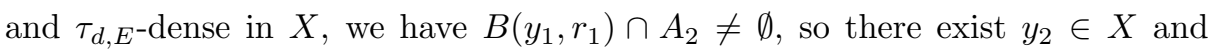


$0<r_{2}<a / 2$ such that $C\left(y_{2}, r_{2}\right) \subset B\left(y_{1}, r_{1}\right) \cap A_{2}$. Proceeding inductively, we see that there exists a sequence $\left(y_{n}\right)$ in $X$ and a sequence $\left(r_{n}\right)$ in $E$ such that $0<r_{n}$ satisfying, for each $n$,

$$
C\left(y_{n+1}, r_{n+1}\right) \subset B\left(y_{n}, r_{n}\right) \cap A_{n+1} \subset C\left(y_{n}, r_{n}\right) \quad \text { and } \quad r_{n}<a / n .
$$

Now the Cantor Intersection Theorem guarantees that $\bigcap_{n=1}^{\infty} C\left(y_{n}, r_{n}\right)$ is a singleton. From $\bigcap_{n=1}^{\infty} C\left(y_{n}, r_{n}\right) \subset B(x, r) \cap A$, we see that $B(x, r) \cap A \neq \emptyset$.

\section{Fixed point theorem}

In this section we will give Banach fixed point theorem on vector metric spaces.

TheOrem 3.1. Let $X$ be an E-complete vector metric space with $E$ is Archimedean. Suppose the mapping $T: X \rightarrow X$ satisfies the contractive condition

$$
d(T x, T y) \leq k d(x, y) \quad \text { for all } x, y \in X,
$$

where $k \in[0,1)$ is a constant. Then $T$ has a unique fixed point in $X$ and for any $x_{0} \in X$, iterative sequence $\left(x_{n}\right)$ defined by $x_{n}=T x_{n-1}$, for $n \in \mathbb{N}, E$-converges to the fixed point of $T$.

Proof. Let $x_{0} \in X$ be arbitrary. Define the sequence $\left(x_{n}\right)$ by $x_{n}=T x_{n-1}$, for $n \in \mathbb{N}$. We have

$$
d\left(x_{n}, x_{n+1}\right)=d\left(T x_{n-1}, T x_{n}\right) \leq k d\left(x_{n-1}, x_{n}\right) \leq \ldots \leq k^{n} d\left(x_{0}, x_{1}\right) .
$$

Thus for $n, p \in \mathbb{N}$

$$
\begin{aligned}
d\left(x_{n}, x_{n+p}\right) & \leq d\left(x_{n}, x_{n+1}\right)+d\left(x_{n+1}, x_{n+2}\right)+\ldots+d\left(x_{n+p-1}, x_{n+p}\right) \\
& \leq\left(k^{n}+k^{n+1}+\cdots+k^{n+p-1}\right) d\left(x_{0}, x_{1}\right) \leq \frac{k^{n+p-1}}{1-k} d\left(x_{0}, x_{1}\right) .
\end{aligned}
$$

Now since $E$ is Archimedean then $\left(x_{n}\right)$ is an $E$-Cauchy. By the $E$-completeness of $X$, there is $z \in X$ such that $x_{n} \stackrel{d, E}{\longrightarrow} z$. Hence there exists $\left(a_{n}\right)$ in $E$ such that $a_{n} \downarrow 0$ and $d\left(x_{n}, z\right) \leq a_{n}$. Since

$$
\begin{aligned}
d(T z, z) & \leq d\left(T x_{n}, T z\right)+d\left(T x_{n}, z\right) \\
& \leq k d\left(x_{n}, z\right)+d\left(x_{n+1}, z\right) \leq k a_{n}+a_{n+1} \leq(k+1) a_{n},
\end{aligned}
$$

then $d(T z, z)=0$, i.e. $T z=z$. The uniqueness of fixed point is easily seen.

We can prove the following theorem as above. We omit the straightforward proof. 
TheOREM 3.2. Let $X$ be an E-complete vector metric space with $E$ is Archimedean. Suppose the mapping $T: X \rightarrow X$ satisfies the contractive condition

$$
d(T x, T y) \leq a d(x, T x)+b d(y, T y)+c d(x, T y)+e d(y, T x)+f d(x, y)
$$

for all $x, y \in X$, where $a, b, c$, e and $f$ are nonnegative and $a+b+c+e+f<1$. Then $T$ has a unique fixed point in $X$ and for any $x_{0} \in X$, iterative sequence $\left(x_{n}\right)$ defined by $x_{n}=T x_{n-1}$, for $n \in \mathbb{N}, E$-converges to the fixed point of $T$.

Now we give an example.

ExAmPLE 3.3. Let $E=\mathbb{R}^{2}$ with coordinatwise ordering (since $\mathbb{R}^{2}$ is not Archimedean with lexicographical ordering, then we can not use this ordering). As in [3] let

$$
X=\left\{(x, 0) \in \mathbb{R}^{2}: 0 \leq x \leq 1\right\} \cup\left\{(0, x) \in \mathbb{R}^{2}: 0 \leq x \leq 1\right\} .
$$

The mapping $d: X \times X \rightarrow E$ is defined by

$$
\begin{aligned}
& d((x, 0),(y, 0))=\left(\frac{4}{3}|x-y|,|x-y|\right), \\
& d((0, x),(0, y))=\left(|x-y|, \frac{2}{3}|x-y|\right), \\
& d((x, 0),(0, y))=\left(\frac{4}{3} x+y, x+\frac{2}{3} y\right) .
\end{aligned}
$$

Then $X$ is $E$-complete vector metric space.

Let $T: X \rightarrow X$ with $T((x, 0))=(0, x)$ and $T((0, x))=(x / 2,0)$, then $T$ satisfies the inequality (3.1) with $k=3 / 4$. According to Theorem 3.1, $T$ has a unique fixed point in $X$. But $T$ is not a contractive mapping in real valued metric on $X$, thus we can not apply the Banach fixed point theorem on metric space to this example.

REMARK 3.4. The fixed point results of this paper generalize the fixed point theorems in metric spaces to vector metric spaces. Also if $X=E$ and $d$ is absolute valued vector metric, then we obtain fixed point theorems of Riesz space $E$.

\section{REFERENCES}

[1] C. D. Aliprantis and K. C. Border, Infinite Dimensional Analysis, Springer-Verlag, Berlin, 1999.

[2] G. E. Hardy and T. D. Rogers, A generalization of a fixed point theorem of Reich, Canad. Math. Bull. 16 (1973), 201-206.

[3] L.-G. HUANG AND X. ZHANG, Cone metric spaces and fixed point theorems of contractive mappings, J. Math. Anal. Appl. 332 (2007), 1468-1476. 
[4] B. E. Rhoddes, A comparison of various definitions of contractive mappings, Trans. Amer. Math. Soc. 226 (1977), 257-290.

[5] P. P. ZABreǏKo, $K$-metric and $K$-normed linear spaces: survey, Collect. Math. 48 (1997), 825-859.

Manuscript received January 7, 2008

\section{CüneYt ÇEviK}

Depatment of Mathematics

Faculty of Science and Arts

Gazi University

Teknikokullar

06500 Ankara, TURKEY

E-mail address: ccevik@gazi.edu.tr

\section{ISHAK ALTuN}

Depatment of Mathematics

Faculty of Science and Arts

Kirikkale University

71450 Yahsihan Kirikkale, TURKEY

E-mail address: ialtun@kku.edu.tr, ishakaltun@yahoo.com 